\title{
Calidad de la atención médica y adherencia a la guía de manejo colombiana de neumonia adquirida en la comunidad en el Hospital Universitario de Santander: periodo 2014 - 2015
}

\author{
Luis Ernesto Téllez* \\ Sergio Alejandro Gómez-Ochoa** \\ Nicolás Andrés García-Rueda** \\ Daniela Beleño-Payares*** \\ José Luis Osma ****
}

\begin{abstract}
*Médico. Neumólogo. Miembro del Grupo para la Renovación Educativa de la Medicina Interna - GERMINA. Profesor de cátedra. Departamento de Medicina interna. Escuela de Medicina. Facultad de salud. Universidad Industrial de Santander. Bucaramanga. Santander. Colombia. **Estudiante de XII nivel. Escuela de Medicina. Facultad de salud. Universidad Industrial de Santander. Bucaramanga. Santander. Colombia. ***Estudiante de X nivel. Escuela de Medicina. Facultad de salud. Universidad Industrial de Santander. Bucaramanga. Santander. Colombia. ****Médico. Ortopedista. Msc en Epidemiología. Líder del grupo de Investigación en Cirugía y Especialidades - GRICES-UIS. Profesor de cátedra. Departamento de Ortopedia. Escuela de Medicina. Facultad de salud. Universidad Industrial de Santander. Bucaramanga. Santander. Colombia. Correspondencia: Sr. Nicolás García, M.D. Carrera 54 \# 73 - 85. Barrio Lagos del Cacique, Bucaramanga, Santander, Colombia. Teléfono: (+57) 3166280041. Correo: nicolasgarciarueda@gmail.com
\end{abstract}

Resumen

Introducción: la neumonía adquirida en la comunidad es una infección aguda del parénquima pulmonar que corresponde a una importante causa de morbi-mortalidad en Colombia. En esta entidad, se ha asociado la pobre calidad en la atención y la mala adherencia a las guías de manejo con un impacto negativo tanto en el desenlace clínico del paciente, como en la academia y solvencia económica de un Hospital Universitario. Objetivo: describir la calidad de la atención médica y adherencia a la guía colombiana de manejo de la neumonía adquirida en la comunidad en el Hospital Universitario de Santander durante el periodo 2014-2015. Materiales y métodos: estudio descriptivo de corte transversal; se seleccionaron 121 historias clínicas con diagnóstico de Neumonía Adquirida en la Comunidad en el Hospital Universitario de Santander durante el periodo 2014-2015. A partir de estas, se recolectaron variables respecto a la aproximación diagnóstica, asignación de tratamiento y recomendaciones generales, evaluando por cada ítem individual el porcentaje de adherencia a la guía de Neumonía adquirida en la comunidad de la Asociación Colombiana de Infectología y los criterios de calidad en la atención de la Community Acquired Pneumonia Organization. Resultados: los ítems con mayor cumplimiento fueron: el hemoleucograma (100\%) y la toma de radiografía de tórax (98\%). Por otra parte, la clasificación CURB-65 (15\%), la indicación de radiografía de tórax en el control (12\%) y las recomendaciones para cese de tabaquismo y vacunación (0\%) fueron los parámetros con menor rendimiento. Conclusiones: se evidenció una falencia en el proceso de clasificación clínica del paciente, condición que, asociada al escaso uso de laboratorios de diagnóstico microbiológico y el excesivo uso de estudios de imagen no indicados como la tomografía computarizada, favorece un tratamiento ineficiente y el desarrollo de resistencia farmacológica. MÉD.UIS.2019;32(2):23-30

Palabras clave: Neumonía. Guía de Práctica Clínica. Calidad de la Atención de Salud.

\section{Quality of health care and adherence to the Colombian community-acquired pneumonia guideline at the Hospital Universitario de Santander: period 2014 - 2015}

\section{Abstract}

Introduction: community-acquired pneumonia is an acute infection of lung parenchyma, being nowadays an important cause of morbidity and mortality in Colombia. In this entity, poor health care quality and poor adherence to management guidelines has been associated with a negative impact on both, the clinical outcome of the patient and the academic and financial solvency of a university hospital. Objective: 
to describe the quality of medical care and adherence to the Colombian community-acquired pneumonia guideline in the Hospital Universitario de Santander during the 2014-2015 period. Methods: descriptive cross-sectional study. 121 clinical records with diagnosis of community-acquired pneumonia in the Hospital Universitario de Santander were selected. Variables regarding diagnostic approach, treatment assignment and general recommendations were collected from these, evaluating for each item the proportion of adherence to the guideline as well as the quality criteria in medical care defined by the Community Acquired Pneumonia Organization. Results: items with best reported adherence were: complete blood count (100\%) and chest X-ray (98\%). On the other hand, CURB-65 classification (15\%), chest X-ray indicated for the ambulatory control (12\%), as well as recommendations for cessation of smoking and vaccination (0\%), were the parameters with the lowest performance. Conclusions: there was evidence of failure in the clinical classification of the patient, which was associated with poor use of microbiological diagnostic laboratories and overuse of imaging studies such as computed tomography, thus favoring inefficient treatment and development of bacterial pharmacological resistance. MÉD.UIS.2019;32(2):23-30

Keywords: Pneumonia. Guideline Adherence. Quality of Health Care.

¿Cómo citar este artículo?: Téllez LE, Gómez-Ochoa SA, García-Rueda NA, Beleño-Payares D, Osma JL. Calidad de la atención médica y adherencia a la guía de manejo colombiana de neumonía adquirida en la comunidad en el Hospital Universitario de Santander: periodo 2014 - 2015. MÉD. UIS.2019;32(2):23-30. doi: 10.18273/revmed.v32n2-2019003

\section{Introducción}

La Neumonía Adquirida en la Comunidad (NAC) es una infección aguda que afecta el parénquima pulmonar cuya adquisición ocurre en un entorno comunitario, es decir, fuera del ámbito hospitalario o cuya sintomatología inicia durante las primeras 48 horas tras su ingreso, teniendo en cuenta que no debe existir un contacto con centros de salud, hogares geriátricos o cuidados médicos en casa'. En América del Norte se estima que la incidencia de esta condición se encuentra entre 5,16 y 7,06 casos por 1000 personas/año ${ }^{2,3}$, y en los casos que requieren hospitalización, la mortalidad es de aproximadamente el 12\%2,4. Aunque en Colombia no existe un sistema de vigilancia y registro nacional que permita cuantificar de manera precisa su efecto, aproximadamente el $4 \%$ de los egresos hospitalarios corresponden a esta enfermedad con una mortalidad del $9,5 \%$ en pacientes que son hospitalizados 5 . En un estudio realizado en la ciudad de Bucaramanga, también se demuestra la alta morbilidad atribuible a la NAC, ocupando el segundo lugar en frecuencia de las infecciones que obligan a la admisión a Unidades de Cuidados Intensivos ${ }^{6}$. Nacionalmente, la NAC es la primera causa de mortalidad por enfermedades infecciosas y la sexta causa de mortalidad en general, evidenciándose la necesidad de intervenciones que reduzcan el impacto de esta entidad en la salud del país.

Desde el punto de vista clínico, se debe hacer uso de herramientas que faciliten el diagnóstico oportuno y tratamiento óptimo de acuerdo a la evidencia científica ${ }^{5}$, razón por la cual, la implementación de guías de práctica clínica y estándares de calidad de atención médica contribuye a este propósito, pues, la adherencia a las guías de manejo y protocolos, desempeña un papel fundamental en el pronóstico del paciente ${ }^{7}$.

Teniendo en cuenta lo anterior, se define "calidad de la atención médica" como el grado en el que los servicios de salud favorecen un incremento en la posibilidad de desenlaces positivos y deseables para los pacientes de manera coherente y basándose en el conocimiento científico actual ${ }^{8}$. A partir de esto se creó la Organización de Neumonía Adquirida en la Comunidad (CAPO, Community Acquired Pneumonia Organization), cuyo principal objetivo es determinar el grado de adherencia a los criterios de calidad establecidos (uso de criterios diagnósticos específicos, indicaciones para admisión hospitalaria, utilización de ayudas para el diagnóstico microbiológico, etc.) en las instituciones prestadoras de salud para esta enfermedad ${ }^{9}$. De manera similar, la Asociación Colombiana de Infectología elaboró la Guía de Práctica Clínica sobre recomendaciones para el diagnóstico, tratamiento y prevención de la NAC con la finalidad de unificar conceptos y consensuar las conductas médicas para el manejo de esta entidad en Colombia 5 .

A pesar de la relevancia de los parámetros mencionados, al día de hoy no existe en Colombia un registro que evalúe los indicadores de calidad para la atención de la CAPO ni la adherencia a la guía nacional. Esta realidad, también aplica para el Hospital Universitario de Santander (HUS), por lo que se desconoce el grado de cumplimiento de los estándares de calidad y de esta guía en la institución ${ }^{5}$. A partir de esta necesidad, se planteó el presente proyecto de investigación, el cual tiene como objetivo describir tanto la calidad de la atención 
médica de acuerdo a los parámetros de la CAPO como la adherencia a la guía de manejo colombiana de NAC en el HUS durante el periodo 2014-2015.

\section{Materiales y métodos}

El presente proyecto fue avalado por el comité de ética del HUS, como un estudio descriptivo de corte transversal, el cual fue llevado a cabo en el HUS a partir de la revisión de las historias clínicas electrónicas con diagnóstico de egreso de NAC registradas entre el 1 de enero de 2014 y el 31 de diciembre de 2015. Para la identificación de las historias se utilizaron los siguientes códigos de la Clasificación Internacional de Enfermedades, décima edición (CIE10): J159 (neumonía bacteriana no especificada), J189 (neumonía no especificada), J158 (otras neumonías bacterianas), J180 (bronconeumonía no especificada), J851 (absceso del pulmón con neumonía) y $\mathbf{1 7 8} 8$ (neumonía en otras enfermedades clasificadas en otra parte). Se incluyeron únicamente los pacientes mayores de 18 años que requirieron hospitalización ya sea por la severidad del cuadro clínico o por la presencia de los siguientes factores de riesgo social, evaluados por la guía de práctica clínica: incapacidad de autocuidado, alteración mental o cognitiva, incapacidad para mantener la ingestión oral, historia de abuso de sustancias psicoactivas o mal soporte familiar y social. Por otra parte, fueron excluidos aquellos pacientes en los que se evidenció un error en la codificación dada por incompatibilidad entre la conclusión dada por el médico y el código CIE10, además de aquellos con diagnóstico de neumonía asociada al cuidado de la salud o con infección por el Virus de la Inmunodeficiencia Humana y Enfermedad Pulmonar Obstructiva Crónica, estos dos últimos debido a las diferencias en el abordaje de la NAC en presencia de estas patologías. Además, los pacientes cuyas historias clínicas presentaran datos incompletos respecto a su evolución o no fuera posible acceder a las mismas, fueron excluidos.

Se recolectaron variables involucradas tanto en la calidad en la atención de NAC definidas por la CAPO, como la adherencia a la guía de manejo colombiana de $N A C^{(5)}$, siendo estas comunes en las áreas de: diagnóstico clínico y radiológico, clasificación CURB-65 (o CRB-65) para definir necesidad de hospitalización, oximetría de pulso, exámenes y paraclínicos complementarios (radiografía de tórax, exámenes de identificación microbiológica de esputo como el gram, cultivo y tinción de ZiehlNeelsen), tratamiento antibiótico (tiempo para el inicio de la terapia antibiótica, antibióticos utilizados, cambio a vía oral y duración), cambio en la vía de administración, vacunación, evaluación de tabaquismo, satisfacción del paciente y seguimiento clínico y radiológico Al evaluar el cumplimiento de las variables se tomó como un nivel de desempeño óptimo cuando la adherencia era $>90 \%$, intermedio entre 60 - $90 \%$ y bajo $<60 \%{ }^{10}$.

Para afrontar el sesgo de selección se incluyeron todos los posibles códigos del $\mathrm{CIE}-10$ relacionados con el diagnóstico de NAC. Respecto a la recolección de las variables, los encuestadores fueron entrenados en cuanto al manejo del instrumento de recolección, el conocimiento de las guías y estándares de calidad. Seguidamente, se realizó una prueba piloto, procediendo posteriormente a la recolección de la información aplicando una doble digitación de datos para evitar sesgos de información durante la misma.

El registro y análisis se realizó en el programa estadístico STATA $13^{\circledR}$, las variables cualitativas fueron presentadas en proporciones y las cuantitativas en medias y sus respectivos rangos de valores. Se evaluó en cada historia el cumplimiento global e individual de cada una de las variables, presentándose este valor en proporciones, en donde el numerador fue el número de veces que se cumplió con el indicador, y el denominador el número que debió haber cumplido, siendo para este estudio el número de casos. Finalmente, el diseño y construcción de este artículo tuvo en cuenta los parámetros recomendados por la iniciativa Strengthening the Reporting of Observational studies in Epidemiology (STROBE).

\section{Resultados}

\section{Características de la población}

Fueron evaluados 121 casos de NAC en total, 81 $(66,9 \%)$ del año 2014 y 40 (33,1\%) del 2015, de los cuales 78 (64,4\%) fueron hombres, encontrando un promedio de edad de 53 años con un rango entre 18 y 100 años. La gran mayoría eran provenientes del Departamento de Santander (93,6\%) y de zonas urbanas (82,6\%).

Con respecto a los factores de riesgo social $(n=45)$ destacó la incapacidad de autocuidado (60\%) y las alteraciones cognitivas o mentales (55\%), evidenciándose estas predominantemente en los adultos menores de 60 años con 22 casos $(68,9 \%)$. 
Por su parte, las comorbilidades se presentaron principalmente en los individuos mayores de 60 años, siendo la Diabetes Mellitus la más prevalente con 22 casos $(18,2 \%)$ (Ver tabla 1$)$.

\section{Diagnóstico y clasificación}

El cumplimiento de los parámetros de calidad acorde a la guía colombiana de NAC fue variable.
Por ejemplo, desde el punto de vista del diagnóstico se obtuvo un cumplimiento igual o superior al $85 \%$ en todos los parámetros evaluados, sin embargo, destacó el pobre uso de la clasificación CURB-65, la cual se realizó solo en $15 \%$ de los casos de manera global, 22,5\% durante el año 2015 y 11\% durante el 2014 (Ver Tabla 2). La clasificación simplificada CRB-65 se utilizó en menos del 1\% de los pacientes y en ningún caso se usó el Pneumonia Severity Index (PSI).

Tabla 1. Factores de riesgo social y comorbilidades más frecuentes según grupo etario

\begin{tabular}{|c|c|c|c|c|c|c|}
\hline Edad & n (\%) & Factores de riesgo social & ICC & IRC & DM & ECV \\
\hline $10-20$ & $13(10,7 \%)$ & $7(5,8 \%)$ & 0 & 0 & 0 & 1 \\
\hline $21-30$ & $15(12,4 \%)$ & $8(6,6 \%)$ & 0 & 0 & 0 & 0 \\
\hline $31-40$ & $9(7,4 \%)$ & $2(1,7 \%)$ & 1 & 0 & 2 & 1 \\
\hline $41-50$ & $16(13,2 \%)$ & $5(4,1 \%)$ & 1 & 2 & 3 & 0 \\
\hline $51-60$ & $22(18,2 \%)$ & $9(7,4 \%)$ & 1 & 2 & 3 & 2 \\
\hline $61-70$ & $15(12,4 \%)$ & $2(1,7 \%)$ & 2 & 4 & 9 & 1 \\
\hline $71-80$ & $12(10 \%)$ & $4(3,3 \%)$ & 2 & 1 & 2 & 1 \\
\hline $81-90$ & $13(10,7 \%)$ & $4(3,3 \%)$ & 4 & 3 & 3 & 2 \\
\hline $91-100$ & $6(5 \%)$ & $4(3,3 \%)$ & 2 & 0 & 0 & 1 \\
\hline Total & $121(100 \%)$ & $45(37,2 \%)$ & $13(10,7 \%)$ & $12(10 \%)$ & $22(18,2 \%)$ & $9(7,4 \%)$ \\
\hline
\end{tabular}

ICC: Insuficiencia Cardiaca Congestiva, IRC: Insuficiencia Renal Crónica, DM: Diabetes Mellitus, ECV: Enfermedad Cerebrovascular. Fuente: autores.

\section{Paraclínicos complementarios}

Respecto a los laboratorios complementarios sugeridos por la guía para los pacientes hospitalizados con NAC, en el $100 \%$ de los casos se solicitó el hemograma, seguido en frecuencia de la radiografía de tórax (98\%). Por el contrario, la procalcitonina fue solicitada sólo en un $5 \%$ de los casos. En cuanto al estudio microbiológico, ninguna prueba superó el $70 \%$ de solicitudes y en ningún caso se solicitaron exámenes para gérmenes atípicos, ni antígenos urinarios de Legionella spp o Streptococcus spp (Ver Tabla 2).

En relación con otros exámenes, la toracentesis estuvo indicada en 27 casos, pero fue solicitada únicamente en 20 de estos casos (74\%), realizándose estudios microbiológicos y citoquímicos en 17 (85\%) y 16 casos (80\%), respectivamente. De manera similar, la fibrobroncoscopia se realizó en 12 de los 14 casos en que estuvo indicada ( $85.7 \%$ ), mientras que se solicitaron los gases venosos centrales en tan solo 5 casos del total (4\%).
Por otra parte, aunque la tomografía computarizada (TC) de tórax no tiene una indicación clara en la guía nacional, fue uno de los exámenes más solicitados, realizándose en 96 casos ( $79 \%$ del total), siendo su variante de alta resolución la más utilizada (41\%) (Ver Tabla 2).

\section{Tratamiento antibiótico}

En la mayoría de los casos (76\%) el tratamiento antibiótico fue instaurado dentro de las primeras ocho horas y en aquellos que tuvieron clasificación CURB hubo concordancia antibiótica según la guía nacional en 14 casos (12\%). Solo en 21 casos (17\% del total) se realizó paso de antibiótico a la vía oral; no obstante, de los 100 pacientes en quienes no se realizó este cambio, esta decisión fue bien justificada, según los criterios de la guía de práctica clínica, en $67 \%$ de ellos.

Los antibióticos empíricos más frecuentemente empleados fueron: claritromicina (55\%), ampicilina sulbactam (43\%), piperacilina tazobactam (36\%), 
vancomicina (11\%) y ceftriaxona (9\%), sin tener una indicación de acuerdo a la guía en la mayoría de los casos (Ver Tabla 3).

\section{Tabaquismo, control ambulatorio y vacunación}

El tabaquismo fue indagado en 55 casos (45\%) sin recomendarse estrategias para el cese de esta actividad en ningún caso. De los 58 pacientes que tuvieron evolución satisfactoria y cuyo egreso post-tratamiento se dio en la misma institución a 37 (64\%) se les asignó control ambulatorio y sólo a 7 de ellos (12\%) indicación de radiografía de control. En ningún caso hubo un registro acerca de la indicación o recomendación de vacunación para influenza o Streptococcus pneumoniae, ni se realizó encuesta de satisfacción.

Tabla 2. Cumplimiento global de las variables de adherencia a la guía y calidad en la atención

\begin{tabular}{|c|c|c|c|c|c|c|}
\hline Indicadores & \multicolumn{2}{|c|}{ Items } & 2014 & 2015 & Total & $\begin{array}{l}\text { Nivel de } \\
\text { adherencia } \\
\text { total }\end{array}$ \\
\hline \multirow{5}{*}{$\begin{array}{l}\text { Diagnóstico } \\
\text { clínico }\end{array}$} & \multicolumn{2}{|c|}{ Clínica del TRI } & $74(91 \%)$ & $36(90 \%)$ & $110(91 \%)$ & Óptimo \\
\hline & \multicolumn{2}{|c|}{ Clínica sistémica } & $74(91 \%)$ & $33(83 \%)$ & $107(88,4 \%)$ & Óptimo \\
\hline & \multicolumn{2}{|c|}{ Toma de Rx de tórax } & $79(97 \%)$ & $40(100 \%)$ & $119(98 \%)$ & Óptimo \\
\hline & \multicolumn{2}{|c|}{ Criterio radiológico de NAC } & $70(86 \%)$ & $33(83 \%)$ & $103(85 \%)$ & Óptimo \\
\hline & \multicolumn{2}{|l|}{ CURB-65 } & $9(11 \%)$ & $9(23 \%)$ & $18(15 \%)$ & Bajo \\
\hline \multirow{4}{*}{$\begin{array}{l}\text { Diagnóstico } \\
\text { microbiológico }\end{array}$} & \multicolumn{2}{|c|}{ Gram de esputo } & $36(44,4 \%)$ & $17(42,5 \%)$ & $53(43,8 \%)$ & Bajo \\
\hline & \multicolumn{2}{|c|}{ Cultivo de esputo } & $39(48 \%)$ & $20(50 \%)$ & $59(49 \%)$ & Bajo \\
\hline & \multicolumn{2}{|c|}{ Ziehl-Neelsen } & $52(64 \%)$ & $22(55 \%)$ & $74(61 \%)$ & Intermedio \\
\hline & \multicolumn{2}{|c|}{ Hemocultivos } & $50(62 \%)$ & $32(80 \%)$ & $82(68 \%)$ & Intermedio \\
\hline \multirow{10}{*}{$\begin{array}{l}\text { Paraclínicos } \\
\text { complementarios }\end{array}$} & \multirow{6}{*}{ Grupo II } & Hemograma & $81(100 \%)$ & $40(100 \%)$ & $121(100 \%)$ & Óptimo \\
\hline & & BUN & $75(93 \%)$ & $38(95 \%)$ & $113(93 \%)$ & Óptimo \\
\hline & & Creatinina & $76(94 \%)$ & $38(95 \%)$ & $114(94 \%)$ & Óptimo \\
\hline & & Gases arteriales & $69(85 \%)$ & $37(93 \%)$ & $106(88 \%)$ & Intermedio \\
\hline & & Glucemia & $55(68 \%)$ & $18(45 \%)$ & $73(60 \%)$ & Intermedio \\
\hline & & Procalcitonina & $3(4 \%)$ & $3(8 \%)$ & $6(5 \%)$ & Bajo \\
\hline & \multirow{4}{*}{ Grupo III } & TP y TTP & $52(64 \%)$ & $30(75 \%)$ & $82(68 \%)$ & Intermedio \\
\hline & & Bilirrubina total & $20(25 \%)$ & $16(40 \%)$ & $36(30 \%)$ & Bajo \\
\hline & & $\begin{array}{l}\text { Bilirrubina } \\
\text { directa }\end{array}$ & $22(27 \%)$ & $16(40 \%)$ & $38(31 \%)$ & Bajo \\
\hline & & Lactato & $65(80 \%)$ & $30(75 \%)$ & $95(79 \%)$ & Intermedio \\
\hline \multirow{4}{*}{$\begin{array}{l}\text { Uso de } \\
\text { Tomografía } \\
\text { Computarizada }\end{array}$} & \multicolumn{2}{|l|}{$\mathrm{TC}$} & $61(75 \%)$ & $35(88 \%)$ & $96(79 \%)$ & N.A. \\
\hline & \multicolumn{2}{|c|}{ TC Simple } & $18(30 \%)$ & $17(49 \%)$ & $35(36 \%)$ & N.A. \\
\hline & \multicolumn{2}{|c|}{ TC Contrastado } & $15(25 \%)$ & $7(20 \%)$ & $22(23 \%)$ & N.A. \\
\hline & \multicolumn{2}{|l|}{ TCAR } & $28(46 \%)$ & $11(31 \%)$ & $39(41 \%)$ & N.A. \\
\hline \multirow{2}{*}{$\begin{array}{l}\text { Tratamiento } \\
\text { antibiótico }\end{array}$} & \multicolumn{2}{|c|}{ Inicio en las primeras 8 horas } & $59(73 \%)$ & $33(83 \%)$ & $92(76 \%)$ & Intermedio \\
\hline & \multicolumn{2}{|c|}{ Concordancia antibiótica } & $8(10 \%)$ & $6(15 \%)$ & $14(12 \%)$ & Bajo \\
\hline \multirow{2}{*}{$\begin{array}{l}\text { Cambio } \\
\text { antibiótico a vía } \\
\text { oral }\end{array}$} & \multicolumn{2}{|c|}{ Cambio de vía } & $14(17 \%)$ & $7(18 \%)$ & $21(17 \%)$ & Bajo \\
\hline & \multicolumn{2}{|c|}{ Decisión de cambio justificada } & $53(65 \%)$ & $35(88 \%)$ & $88(73 \%)$ & Intermedio \\
\hline Tabaquismo & \multicolumn{2}{|c|}{ Indagación de tabaquismo } & $30(37 \%)$ & $25(63 \%)$ & $55(45 \%)$ & Bajo \\
\hline \multirow{2}{*}{$\begin{array}{l}\text { Control } \\
\text { ambulatorio }\end{array}$} & \multicolumn{2}{|c|}{ Programación de cita de control } & $27(63 \%)$ & $10(67 \%)$ & $37(64 \%)$ & Intermedio \\
\hline & \multicolumn{2}{|c|}{ Indicación de radiografía de control } & $5(12 \%)$ & $2(13 \%)$ & $7(12 \%)$ & Bajo \\
\hline
\end{tabular}

TRI: Tracto respiratorio inferior, Rx: Radiografía, TP: Tiempo de protrombina parcial, TTP: Tiempo de tromboplastina, TC: Tomografía computarizada, TCAR: Tomografía computarizada de alta resolución

Fuente: autores. 
Tabla 3. Uso de antimicrobianos y su concordancia de acuerdo a la guía

\begin{tabular}{|c|c|c|}
\hline Antimicrobiano & $\begin{array}{c}\text { Frecuencia de } \\
\text { uso }\end{array}$ & $\begin{array}{c}\text { Concordancia de } \\
\text { acuerdo a la guía }\end{array}$ \\
\hline Claritromicina & $67(55 \%)$ & $11(16 \%)$ \\
\hline $\begin{array}{c}\text { Ampicilina- } \\
\text { sulbactam }\end{array}$ & $52(43 \%)$ & $5(10 \%)$ \\
\hline $\begin{array}{c}\text { Piperacilina- } \\
\text { tazobactam }\end{array}$ & $43(36 \%)$ & $2(5 \%)$ \\
\hline Vancomicina & $13(11 \%)$ & $5(38 \%)$ \\
\hline Ceftriaxona & $11(9 \%)$ & $1(9 \%)$ \\
\hline Meropenem & $4(3 \%)$ & $0(0 \%)$ \\
\hline Linezolid & $3(3 \%)$ & $0(0 \%)$ \\
\hline Clindamicina & $2(2 \%)$ & $0(0 \%)$ \\
\hline Doxiciclina & $2(2 \%)$ & $0(0 \%)$ \\
\hline Oseltamivir & $5(4 \%)$ & $0(0 \%)$ \\
\hline
\end{tabular}

Fuente: autores.

\section{Discusión}

\section{Diagnóstico}

En el presente estudio, se evidenció un cumplimiento variable de los componentes de la guía nacional destacando en primera instancia el diagnóstico clínico, el cual se recomienda que se realice a partir de tres aspectos clave: síntomas del tracto respiratorio inferior, síntomas sistémicos y evidencia radiológica del compromiso infeccioso. Respecto a esto se encontró un cumplimiento aceptable de estos indicadores siendo el ítem con menor rendimiento el criterio radiológico, el cual se clasificó en un nivel intermedio (85\%), aunque no siempre se correlacionó con la clínica; como en un caso en que se evidenció ausencia de sintomatología sistémica y respiratoria, pero se realizó el diagnóstico únicamente con el criterio radiológico. Al comparar el resultado del parámetro radiológico con otros estudios similares en el país como el de Machado-Alba JE et al y Martínez C et al., realizados en ciudades como Bogotá, Medellín, Pereira y Neiva se evidenciaron cifras semejantes de cumplimiento con $74,7 \%$ y $95 \%$ respectivamente ${ }^{11,12}$.

Por otra parte, la TC no tiene indicación según las recomendaciones de la guía, hecho que contrasta con el $79 \%$ de solicitudes en este estudio, una incidencia mucho mayor a la esperada, representando un sobrecosto de la atención.

\section{Grado de complejidad del tratamiento (Clasificación CURB-65)}

Se recomienda que se realice una clasificación de la severidad clínica del paciente a través de escalas validadas tales como el CURB-65, su versión abreviada CRB-65 o el PSI, pues son herramientas que permiten estimar la mortalidad y garantizar una adecuada asignación del tratamiento. En este estudio se reporta una pobre aplicación de estas con un cumplimiento general de $15 \%$ para todos los índices, siendo el más utilizado el CURB-65, condición que evidenció una mejoría relativa en el 2015 lo que podría reflejar un incremento en el conocimiento de la guía a medida que transcurre el tiempo desde su publicación. No obstante, este incremento es insuficiente, por lo que existe la necesidad de mejorar el proceso de difusión de la guía y que este se haga de manera activa y sistemática. Cuando se comparó el rendimiento de este ítem con otros estudios a nivel internacional se observó un cumplimiento variable, encontrando proporciones de uso desde $2,3 \%$ hasta $79,5 \% 13,14$, mientras que, en otros estudios similares a pesar de haber calculado la escala a partir de la historia clínica, refieren la falta de realización y registro de estas herramientas en los servicios de urgencias sin señalar una proporción específica de uso $^{15,16}$. Lamentablemente, los estudios nacionales relativos a este tema fueron realizados en años anteriores a la aparición de estas herramientas o no las tuvieron en cuenta.

La guía nacional recomienda determinados laboratorios complementarios en base a la severidad del cuadro y la clasificación clínica CURB-65: a los pacientes clasificados en el grupo II se les deben solicitar laboratorios analíticos básicos, mientras que los clasificados en el grupo III requieren de exámenes más especializados (Ver Tabla 2). Debido a que la clasificación CURB-65 no se utilizó en la mayoría de pacientes, en general no fue posible determinar a qué pacientes se les debió haber solicitado exámenes del grupo III. Sin embargo, sí se puede evidenciar que los laboratorios básicos sugeridos por la guía (los correspondientes al grupo II) fueron solicitados en la mayoría de casos (Ver Tabla 2). No obstante, es de resaltar que existe una falencia relativa en los laboratorios de diagnóstico microbiológico, dado que sólo en un $43,8 \%$ de los pacientes se realizaron los tres estudios sugeridos de esputo: gram, cultivo y baciloscopia; sin embargo, la investigación de otros gérmenes no se solicitó en ningún paciente. 
Además, la guía sugiere el uso de la procalcitonina como método de seguimiento de la respuesta clínica en cuanto al tratamiento antibiótico, utilizándose esta únicamente en el $5 \%$ de los casos a pesar de estar disponible en la institución. El porcentaje de adherencia a la realización de radiografía de tórax, hemocultivos y hemograma, fue superior al ser comparado con el estudio de Machado et al., el cual reportó una tasa de cumplimiento de $74,7 \%, 31 \%$ y $82,5 \%$, respectivamente ${ }^{11}$.

\section{Tratamiento}

Según el protocolo de la CAPO un indicador de calidad corresponde a la oportunidad para el tratamiento antibiótico dentro de las primeras ocho horas desde el ingreso del paciente lo cual se cumplió en el $76 \%$ de los casos, hallazgo que, aunque no se ubica en el nivel bajo, fue inferior al del estudio de Christensen et al. con una adherencia del $85 \%{ }^{10}$. Por otra parte debido a que la mayoría de los pacientes no fueron clasificados no fue posible valorar en ellos si hubo concordancia antibiótica, por lo tanto, se considera que no hubo adherencia a la guía en este aspecto. Adicionalmente, en los pacientes en los que se aplicó la clasificación CURB-65 solo un $12 \%$ siguió las recomendaciones de la guía para el esquema antibiótico. A este respecto se desconoce por tanto qué criterio o instrumento se utilizó para la asignación del tratamiento, pues resulta preocupante que en promedio el rendimiento de los indicadores de diagnóstico microbiológico fue bajo (55\%). Este hecho resulta relevante debido a que la asignación no justificada de antibioticoterapia y el uso indiscriminado de antibióticos de amplio espectro determina un incremento de los costos, aumento de la resistencia bacteriana y un ejercicio académico inadecuado en el contexto de un hospital universitario; un claro ejemplo de este aparente uso indiscriminado lo constituye la alta frecuencia de aplicación de la piperacilina-tazobactam en un 36\%, sólo estando indicado por la presencia de factores de riesgo para Pseudomonas aeruginosa en un 5\% de las ocasiones en las que fue aplicado. De manera similar, la ceftriaxona se ordenó como tratamiento en un $9 \%$ de los casos y en sólo un caso fue correcta su indicación, dada por factores de riesgo para neumococo resistente a la penicilina. Al comparar estos resultados con estudios llevados a cabo en la región, se evidencia en estos un mayor uso de cefalosporinas de tercera generación (17,8\% - 32,6\% de los casos evaluados) y en contraste la ausencia de prescripción de piperacilina-tazobactam. Cabe destacar que en estos estudios no se valoró la pertinencia de dichos antibióticos, por lo que los hallazgos respecto a esto no son comparables ${ }^{11,12}$.

El cambio de vía intravenosa a oral, de acuerdo con la guía, se debe realizar tan pronto se logre mejoría clínica y se cumplan los parámetros allí descritos. Dicho cambio fue realizado en el $17 \%$ de los casos, sin embargo, en la mayoría de aquellos en los que no se realizó, esta decisión estuvo bien justificada debido a que la institución es un hospital de tercer nivel donde las complicaciones, remisiones e incluso decesos son frecuentes. Aun así, para los casos en que no se realizó dicho cambio y no había justificación clara, es importante resaltar que la omisión de esta sugerencia conlleva un mayor riesgo de complicaciones, mayor costo y menor satisfacción para el paciente.

La saturación de oxígeno es una medida que se relaciona directamente con la necesidad del mismo, respecto a esto la guía nacional recomienda un nivel de saturación de oxígeno mayor al 90\%. Se documentó que la oximetría de pulso se realizó al ingreso el en $97 \%$ de los pacientes, hecho que permitió determinar la necesidad de oxigenoterapia en casi la totalidad de los pacientes, siendo superior al estudio de Levy et al, en el cual este parámetro se registró en un $15 \%$ de los $\operatorname{casos}^{13}$.

\section{Prevención: tabaquismo y vacunación}

A pesar de que la guía recomienda la vacunación ( $H$. Influenza y S. pneumoniae) para reducir los eventos infecciosos y la cesación del tabaquismo sobresale la ausencia de cumplimiento de esta estrategia en nuestro medio, pues no existe reporte en la historia clínica de haber realizado esta recomendación, contrario a un estudio similar en el cual se documentó vacunación antineumocóccica en el $51 \%$ de los pacientes, vacuna antigripal en el $82 \%$ y ofrecimiento para abandonar el tabaquismo en el $29 \%^{10}$.

Merece mencionarse también que, aunque la encuesta de satisfacción es un requisito de los organismos de calidad, no es una práctica tenida en cuenta y no se llevó a cabo en ningún paciente.

Se destaca el presente estudio como principal limitación que los datos fueron extraídos de una fuente secundaria, lo cual representa un posible sesgo de información. En cuanto a las fortalezas del trabajo, se trata del primer estudio de este tipo en Colombia, lo que permite evidenciar objetivamente las falencias en el proceso de atención médica en 
cuanto a la calidad de la misma y la adherencia a la guía de manejo nacional, así como la inadecuada racionalización económica del manejo médico en la solicitud de TC y el manejo antibiótico.

\section{Conclusiones}

La aplicación de las guías de manejo impacta positivamente en múltiples áreas. Su aplicación sistemática permite la mejora del proceso educativo, los desenlaces médicos, la calidad de la atención y por tanto aporta a la acreditación institucional y aumenta la racionalización económica en la atención médica, aspectos que son de vital relevancia en el contexto de un hospital universitario. Por tanto, es necesaria una estrategia de difusión de la guía y de los estándares de calidad, priorizando el componente educativo y la institucionalización de lineamientos que garanticen su cumplimiento. Se espera que lo aquí expuesto sirva como fuente de información en el proceso educativo y de esta manera, se pueda orientar la toma de decisiones de forma más apropiada y costoeficiente.

\section{Conflictos de interés}

No existe conflicto de interés alguno por parte de autor y coautores.

\section{Financiación}

El presente estudio no recibió financiación alguna.

\section{Consideraciones éticas}

La investigación se realizó teniendo en cuenta la declaración de Helsinki de la asociación médica mundial y de acuerdo a la resolución 8430 de 1993 del Ministerio de Salud nacional, esta investigación clasifica como "sin riesgo". Por el diseño retrospectivo del estudio no se requirió consentimiento informado por parte de los participantes y se respetó la dignidad y confidencialidad de los mismos ya que no se reportó información que permita identificarlos; de igual manera, bajo ninguna circunstancia se vulneran los principios de beneficiencia, no maleficiencia, respeto y justicia.

\section{Referencias bibliográficas}

1. Ho ED-P. Community-Acquired Pneumonia in Adults and Children. Prim Care Clin Off Pract. 2013 Sep 1;40(3):655-69.

2. Marrie TJ, Huang JQ. Epidemiology of community-acquired pneumonia in Edmonton, Alberta: an emergency departmentbased study. Can Respir J. 2005 Apr;12(3):139-42.

3. Ramirez JA, Wiemken TL, Peyrani P, Arnold FW, Kelley R, Mattingly WA, et al. Adults Hospitalized With Pneumonia in the United States: Incidence, Epidemiology, and Mortality. Clin Infect Dis Off Publ Infect Dis Soc Am. 2017 Nov 13;65(11):1806-12.

4. Kalil AC, Metersky ML, Klompas M, Muscedere J, Sweeney DA, Palmer LB, et al. Management of Adults With Hospital-acquired and Ventilator-associated Pneumonia: 2016 Clinical Practice Guidelines by the Infectious Diseases Society of America and the American Thoracic Society. Clin Infect Dis Off Publ Infect Dis Soc Am. 2016 Sep 1;63(5):e61-111.

5. Recomendaciones para el diagnóstico, tratamiento y prevención de la neumonía adquirida en la comunidad en adultos inmunocompetentes. Infectio. 2013 Mar 1;17:1-38.

6. Carvajal Estupiñán JF, Naranjo Junoy F, Ospina Diaz JM. Caracterización de pacientes diagnosticados con sepsis en una unidad de cuidados intensivos de Bucaramanga, Colombia 2010-2011: estudio descriptivo. Arch Med Manizales. 2016 Jun 30;16(1):53-60.

7. Jaramillo-Jaramillo L, Martínez-Sánchez L, Gaviria-García J. Neumonía adquirida en comunidad: Adherencia a las guías terapéuticas, importante eslabón en el desenlace clínico del paciente. Rev Cient Cienc Med 2015; 18(1): 36-42.

8. Metersky ML, Abend SL, Meehan TP. What have we learned about how to measure quality of care for patients with community-acquired pneumonia? Respir Care Clin N Am. 2005 Mar;11(1):87-98.

9. Ramírez J. An International, Observational Study to Evaluate Current Management of Hospitalized Patients with CommunityAcquired Pneumonia (International CAP Study) Protocol \# 10,000 June 29, 2001 (Protocolo de investigación).

10. Christensen D, Luna C, Martinez J, Rodriguez E, Marzoratti L, Gonzalez J, et al. Cumplimiento con las guías nacionales en pacientes hospitalizados con neumonía adquirida en la comunidad: Resultados del estudio capo en Argentina. MEDICINA (Buenos Aires). 2007; 67: 709-713.

11. Machado-Alba JE, Isaza-Martínez B, Sepulveda-Marín M, Rivera-Palacios A, Ceballos SL. Nivel de adherencia a la guía de atención de neumonía adquirida en la comunidad en el Hospital Universitario San Jorge de Pereira, Colombia. Revista médica Risaralda. 2012;18(2):134-139.

12. Martínez C, Jaimes F, Montúfar F, Hincapié G, Morales A, Acero R, et al. Procesos de atención y prescripción en neumonía adquirida en la comunidad en hospitales universitarios en Colombia. Rev Colomb Neum.2003; 15(4):181-189.

13. Levy G, Perez M, Rodríguez B, Hernández Voth A, Perez J, Gnoni $\mathrm{M}$, et al. Cumplimiento con las guías nacionales en pacientes hospitalizados con neumonía adquirida en la comunidad: resultados del Estudio Capo en Venezuela. Arch Bronconeumol. 2015 Apr 1;51(4):163-8.

14. Al-Abri SS, Al-Maashani S, Memish ZA, Beeching NJ. An audit of inpatient management of community-acquired pneumonia in Oman: a comparison with regional clinical guidelines. J Infect Public Health. 2012;5(3):250-6.

15. Conterno LO, Moraes FY, Silva Filho CR. Implementation of community-acquired pneumonia guidelines at a public hospital in Brazil. J Bras Pneumol. 2011;37(2):152-9.

16. Guo Q, Li HY, Zhou YP, Li M, Chen XK, Liu H, et al. Compliance with the CURB-65 score and the consequences of nonimplementation. Int J Tuberc Lung Dis. 2011;15(12):1697-702. 